\title{
TRITERPENOIDES, FENÓLICOS E EFEITO FITOTÓXICO DAS FOLHAS DE Eugenia flavescens DC (Myrtaceae)
}

\author{
Antonio J. Cantanhede Filho ${ }^{\mathrm{a}, *, \#, \text { Lourivaldo S. Santos }}{ }^{a}$, Giselle M. S. P. Guilhon ${ }^{\mathrm{a}}$, Maria das Graças B. Zoghbi ${ }^{\mathrm{b}}$, Pollyane \\ S. Ports ${ }^{c}$ e Iris C. S. Rodrigues ${ }^{b}$ \\ anstituto de Ciências Exatas e Naturais, Universidade Federal do Pará, 66075-110 Belém - PA, Brasil

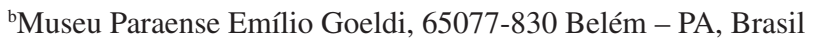 \\ ${ }^{c}$ Faculdade de Engenharia de Alimentos, Instituto de Química, Universidade Estadual de Campinas, 13083-862 Campinas - SP, Brasil
}

Recebido em 19/07/2016; aceito em 07/11/2016; publicado na web em 25/11/2016

\begin{abstract}
TRITERPENOIDS, PHENOLICS AND PHYTOTOXIC EFFECTS FROM Eugenia flavescens DC (MYRTACEAE) LEAVES. This research deals with the chemical study and evaluation of the phytotoxic effects of the methanol extract of E. flavescens leaves. The compounds were isolated using classic chromatographic techniques and the identification of the phenolic profile was proposed from the HPLC/DAD/MS-MS data. Structure elucidation of the compounds was performed by ${ }^{1} \mathrm{H}$ and ${ }^{13} \mathrm{C}(1 \mathrm{D}$ e $2 \mathrm{D}) \mathrm{NMR}$ techniques and HRMS. The chemical study led to the isolation of betulinic acid (1) and lupeol (2), identified in mixture quercitrin (3) and catechin (4) beyond myricetrin (5) in mixture with (3). In addition, several phenolic compounds were identified, among them, flavonoids and phenolic acids. For bioassays phytotoxic effect were used as test plants two invasive species, $M$. pudica and S. obtusifolia. The methanol extract showed significant seed germination inhibition. The triterpenoid 1 showed low inhibition effects on seed germination and moderate effects on radicle and hypocotyl growth. The mixture of flavonoids $3 / 4$ showed effective phytotoxic effects, inhibiting $92,1 \%$ of the seed germination of $M$. pudica and $63,0 \%$ of $S$. obtusifolia. Inhibitions of $72,5 \%$ and $76,1 \%$ of $M$. pudica radicle and hypocotyl growths were observed, respectively, and $74,5 \%$ and $78,0 \%$ of S. obtusifolia for the same bioassays, respectively.
\end{abstract}

Keywords: flavonols; catechin; phenolic acids; invasive species.

\section{INTRODUÇÃO}

A familia Myrtaceae é nativa de regiões tropicais, principalmente América do Sul, Sudeste da Ásia e Austrália, compreende 132 gêneros e 4.620 espécies, sendo que no Brasil há 48 gêneros e mais de 900 espécies. ${ }^{1}$ Dentre os inúmeros gêneros pertencentes a essa família, destaca-se o gênero Eugenia que possui cerca de 500 espécies distribuídas, principalmente em regiões tropicais e subtropicais da América, em regiões tropicais da Ásia e algumas espécies ocorrendo na Austrália e na África. ${ }^{2}$ As espécies pertencentes a esse gênero possuem frutos comestíveis e apresentam atividade anti-inflamatória, analgésica, antipirética e antifúngica. ${ }^{3,4}$

Na literatura, não há trabalhos sobre a composição química de Eugenia flavescens. O óleo essencial das folhas possui como constituintes químicos majoritários os sesquiterpenos $\alpha$-cis-bisaboleno e $\beta$-bisaboleno. ${ }^{5}$

Na busca de bioherbicidas, a espécie E. flavescens foi selecionada para estudo químico e avaliação do potencial fitotóxico associado sobretudo à inibição da germinação de sementes, do crescimento da radícula e do hipocótilo frente a duas espécies de plantas invasoras de pastagens, Mimosa pudica (malícia) e Senna obtusifolia (mata-pasto).

As plantas daninhas, espécies infestantes ou invasoras, que trazem prejuízo às culturas, são um dos fatores mais importantes a impor limitações ao desenvolvimento da atividade agrícola no mundo. ${ }^{6}$ Os efeitos tóxicos dos herbicidas comerciais conhecidos na saúde humana vão desde náuseas e vômitos até alguns tipos de cânceres. Quanto ao meio ambiente, os herbicidas podem acumular-se na biota

*e-mail: prof.antoniofilho@ifma.edu.br

\#Endereço atual: Departamento Acadêmico de Química, Instituto Federal de Educação Ciência e Tecnologia do Maranhão, 65030-005 São Luís - MA, Brasil This paper is part of the PubliSBQ Special Issue in honor of the late Prof. Angelo da Cunha Pinto. ou contaminar a água e o solo, podendo levar ao desequilíbrio ecológico. ${ }^{7}$ Embora não se possa descartar o uso de herbicidas sintéticos completamente, um manejo utilizando potencialidades fitotóxicas pode reduzir seu uso até um ponto que não prejudique a produção agrícola e traga menos danos ao meio ambiente. ${ }^{8}$

\section{PARTE EXPERIMENTAL}

\section{Procedimentos experimentais gerais}

Obtenção do material vegetal e preparação das fases orgânicas As folhas de Eugenia flavescens DC. foram coletadas na praia da Murieta, Reserva Extrativista da Marinha, no município de Maracanã, Pará. A identificação foi feita pelo botâncio Luis Carlos Lobato no herbário do Museu Paraense Emilio Goeldi, (Belém - PA), onde uma exsicata foi depositada sob o registo MG 196794.

As folhas $(5,0 \mathrm{~kg})$, após secagem durante uma semana em ambiente com baixa umidade, foram moídas em moinho de facas e extraídas por maceração durante uma semana com metanol. A solução resultante foi concentrada sob vácuo fornecendo $56,9 \mathrm{~g}$ de extrato bruto metanólico (EBM). Parte do EBM (32,8 g) foi solubilizado em metanol-água destilada $\left(\mathrm{MeOH} / \mathrm{H}_{2} \mathrm{O} 70 \%\right)$ e particionado com hexano, diclorometano, acetato de etila e $n$-butanol. As soluções de cada fase foram concentradas sob vácuo.

\section{Isolamento e purificação}

As fases orgânicas, com exceção da fase $n$-butanol (2,3 g), foram submetidas, separadamente, à cromatografia em coluna (CC) em sílica (70 -230 mesh) utilizando como eluentes misturas de hexano e acetato de etila e acetato de etila e metanol em ordem crescente de polaridade. Sucessivos procedimentos semelhantes ao anterior foram usados na purificação de algumas frações. As frações mais polares foram purificadas por CC utilizando-se como fase estacionária Sephadex LH-20 e metanol como eluente. As frações foram analisadas por cromatografia 
em camada delgada analítica (CCDA) em cromatoplacas de gel de sílica 60 F254 sobre alumínio utilizando-se como revelador uma solução ácida de sulfato cérico, seguida de aquecimento.

O fracionamento da fase hexânica ( $3,8 \mathrm{~g})$ originou 28 frações. Das frações 22-25 eluídas com uma mistura de hexano e acetato de etila (Hex-AcOEt 6:4), após precipitação e lavagem com uma mistura de hexano (Hex) e diclorometano (DCM) Hex-DCM 1:1 à baixa temperatura, foi obtida a substância $1(25 \mathrm{mg})$. A fase diclorometânica (4,3 g) originou 40 frações. A fração 8 eluída com (Hex-AcOEt 8:2), após precipitação e lavagem com a mistura de (Hex-DCM 1:1) à baixa temperatura, forneceu uma quantidade adicional da substância 1 (90 mg), e as frações de 10-20 eluídas com (Hex-AcOEt 6:4), após o mesmo procedimento, forneceram a substância $2(5 \mathrm{mg})$.

As frações 6 e 7 (FA 6 e FA 7, $220 \mathrm{mg}$ ) resultantes do fracionamento inicial por CC em sílica da fase em acetato de etila $(5,7 \mathrm{~g})$, eluição com AcOEt, foram reunidas e submetidas a CC utilizando Sephadex LH-20 como fase estacionária, resultando em 40 frações (designadas frações Spx). A fração Spx 18 foi identificada como uma mistura das substâncias 3 e 4 (22 mg) e a fração Spx 27, uma mistura das substâncias 3 e 5 (28 mg). As frações Spx 11, Spx 14, Spx 21 e Spx 37 foram selecionadas, juntamente com as frações FA 8 (eluída com AcOEt-MeOH 95:5) e FA 12 (eluída com AcOEt-MeOH 9:1), da fase em acetato de etila para verificação do perfil de compostos fenólicos presentes, em que foi possível identificar as substâncias $\mathbf{4}$ e $\mathbf{6}$ a $\mathbf{1 3}$.

\section{Análise por RMN e EMAR}

Os espectros de $\mathrm{RMN}$ de ${ }^{1} \mathrm{H}$ e de ${ }^{13} \mathrm{C}(1 \mathrm{D}$ e $2 \mathrm{D})$ do ácido betulínico (1), lupeol (2) e das misturas de quercitrina (3) com catequina (4), quercitrina (3) com miricitrina (5) foram obtidos em espectrômetro Varian, modelo MERCURY-300 (300 MHz para ${ }^{1} \mathrm{H}$ e $75 \mathrm{MHz}$ para $\left.{ }^{13} \mathrm{C}\right)$, em $\mathrm{C}_{5} \mathrm{D}_{5} \mathrm{~N}(\mathbf{1})$ e $\mathrm{CD}_{3} \mathrm{OD}(\mathbf{2}-\mathbf{5})$ como solventes. Os deslocamentos químicos $(\delta)$ foram registrados em ppm com base na referência interna o sinal do TMS. Os dados obtidos foram comparados com os dados da literatura. Para confirmação da identificação das misturas (3/4) e (3/5) também foi utilizado EMAR em espectrômetro Waters Xevo G2-S Qtof/Tof.

\section{Perfil de fenólicos por CLAE/DAD/EM-EM}

A análise do perfil das frações em acetato de etila foi realizada empregando-se um cromatógrafo líquido de alta eficiência, da marca Thermo, com injetor automático, alça de amostragem de $20 \mu \mathrm{L}$ e bomba quaternária, acoplado a um detector de arranjo de diodos (DAD) e um espectrômetro de massas equipado com fonte de ionização por electrospray (ESI) e analisador de massas Ion-Trap. O equipamento foi operado à temperatura ambiente $\left(25 \pm 2{ }^{\circ} \mathrm{C}\right)$ e os dados cromatográficos foram obtidos e processados pelo software Xcalibur. A coluna cromatográfica utilizada foi do tipo fase reversa, $\mathrm{C}_{18}(150 \mathrm{x}$ $2.1 \mathrm{~mm}$ ) e tamanho de partícula de $1,9 \mu \mathrm{m}$ (Thermo, hypersil gold). $\mathrm{O}$ método cromatográfico foi baseado naquele descrito por Novakova et al., ${ }^{9}$ com pequenas modificações. A fase móvel constituiu de solução aquosa ácido fórmico a $0,1 \%$ (A) e acetonitrila (B). O gradiente de eluição iniciou-se na proporção 95:5 em um fluxo de $0,32 \mathrm{~mL} \mathrm{~min}^{-1}$. A concentração de "A" decresceu para a condição 50:50 e para isto gastou-se o tempo de 5 minutos, a partir dos 6 minutos voltou gradativamente para a condição inicial 95:5, o que demorou o tempo de 20 minutos, e permaneceu nesta condição por mais 2 minutos para que as condições iniciais fossem restabelecidas. A detecção dos compostos foi realizada utilizando detector DAD (operando a 210 , 260, 300 e $325 \mathrm{~nm}$ ) e um espectrômetro de massas com uma fonte de electrospray operando no modo negativo (temperatura do capilar 350 ${ }^{\circ} \mathrm{C}$, voltagem capilar de $2,5 \mathrm{kV}$, tensão do cone de $5 \mathrm{kv}$ ). Gás hélio (He) foi utilizado como gás de colisão e o nitrogênio $\left(\mathrm{N}_{2}\right)$ foi usado como gás nebulizador 70 (unidade arbitraria). Para a identificação dos compostos presentes nas amostras foram utilizados o tempo de retenção, os espectros de absorção e a co-cromatografia comparando com os dos padrões e espectro de massas, que auxiliou na confirmação da estrutura química dos compostos.

\section{Metodologia dos bioensaios}

Os bioensaios para avaliação dos efeitos fitotóxicos foram realizados de acordo com metodologias descritas na literatura. ${ }^{10-12}$ Os testes foram realizados com as fases orgânicas e com as substâncias ácido betulinico (1), quercitrina e catequina em mistura (3/4).

\section{Plantas receptoras}

As sementes das plantas receptoras, $M$. pudica e $S$. obtusifolia, foram coletadas na área de produtores do município de Castanhal (PA), passaram por processo de limpeza e foram tratadas para a quebra de dormência, em imersão em ácido sulfurico $\left(\mathrm{H}_{2} \mathrm{SO}_{4}\right)$. PA, por 15 minutos (malícia) e 20 minutos (mata-pasto), posteriormente foram lavadas em água corrente por 10 minutos e secas à temperatura ambiente. ${ }^{13}$

\section{Germinação}

Foi utilizada câmara de germinação BOD, com temperatura constante de $25^{\circ} \mathrm{C}$ e fotoperíodo de 12 horas. Cada placa de Petri de $9,0 \mathrm{~cm}$ de diâmetro, forrada com papel de filtro qualitativo, recebeu $3 \mathrm{~mL}$ da solução a ser testada, fases orgânicas a $1 \%(\mathrm{~m} / \mathrm{v})$, o ácido betulínico (1) nas concentrções de 200, 400, 600 e 800 ppm e a mistura de flavonoides quercitrina e catequina (3/5) nas concentrações de 50, 75, 100 e 150 ppm, todas em triplicata. Após evaporação do solvente o papel de filtro foi umedecido com uma solução aquosa de fungicida (micostatim-1\%), em seguida foram colocadas 20 sementes das plantas receptoras. As testemunhas receberam apenas a solução fungicida aquosa. Foi considerada semente germinada aquela que apresentava extensão radicular igual ou superior a 2,0 mm. A germinação das sementes foi monitorada em períodos de 4 dias, sendo que 24 horas após a montagem do experimento foi realizada a primeira contagem de germinação, com contagens diárias e eliminação das sementes germinadas. Para o cálculo do percentual de inibição da germinação (I) utilizou-se a Equação 1. ${ }^{10-14}$

$$
\mathrm{I}(\%)=\left[1-\left(\mathrm{SG}_{\text {amostra }}\right)\right] \times 100 / \mathrm{SG}_{\text {controle }}
$$

Em que $\mathrm{SG}_{\text {amostra }}$ é o número de sementes germinadas e $\mathrm{SG}_{\text {controle }}$ é o número de sementes germinadas na amostra controle.

\section{Desenvolvimento da radícula e do hipocótilo}

A avaliação do crescimento de plântulas foi desenvolvida utilizando-se as mesmas condições usadas para a germinação de sementes. Para cada concentração foram colocadas seis sementes pré-germinadas de $S$. obtusifolia e $M$. pudica, com três dias de germinação. Ao final do período de 10 dias de crescimento, mediu-se o comprimento da radícula e do hipocótilo. Para o cálculo do percentual de inibição de crescimento (IC) da radícula e do hipocótilo, utilizou-se a Equação 2.14,15

$$
\mathrm{IC}(\%)=\left[1-\left(\mathrm{CEC}_{\text {amostra }}\right)\right] \times 100 / \mathrm{CEC}_{\text {controle }}
$$

Em que $\mathrm{CEC}_{\text {amostra }}$ é o comprimento (cm) da radícula ou do hipocótilo e $\mathrm{CEC}_{\text {controle }} \mathrm{O}$ comprimento $(\mathrm{cm})$ da radícula ou do hipocótilo na amostra controle.

\section{Análise de dados}

O delineamento experimental foi inteiramente casualizado, com três repetições, para verificação dos efeitos biológicos. Os gráficos 
de barras e de linha de tendência foram feitos no software Excel (Office-2013), assim como as equações quadráticas das curvas, que, por sua vez, foram utilizadas para o cálculo do $\mathrm{IC}_{50}$.

\section{RESULTADOS E DISCUSSÃO}

As substâncias obtidas do extrato metanólico das folhas de E. flavescens foram identificadas como ácido betulínico (1) e lupeol (2), além da identificação das substâncias em mistura quercitrina (3) e catequina (4) e miricitrina 5. As estruturas das substâncias $\mathbf{1}$ - 5 encontram-se na Figura 1.

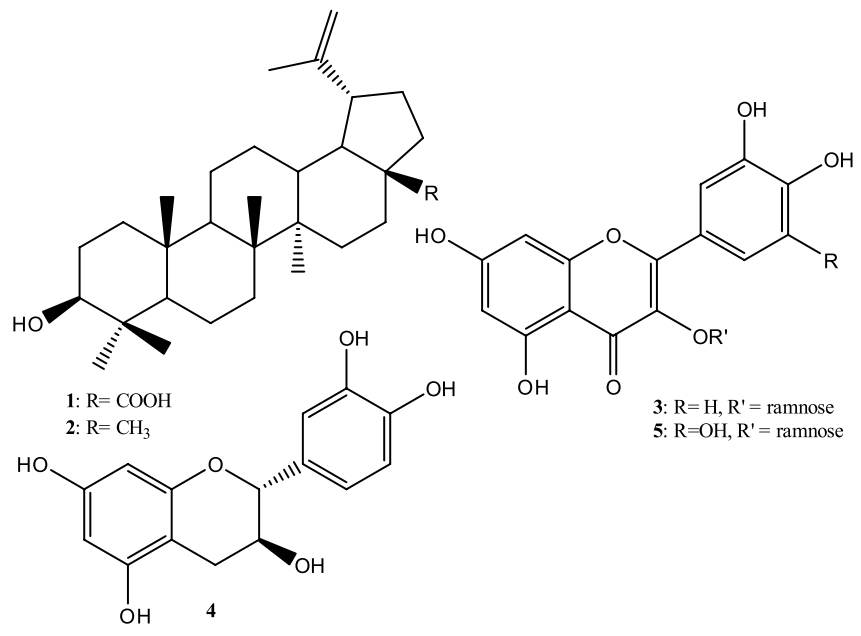

Figura 1. Substâncias identificadas nas folhas de E. flavescens

Substância 1: RMN de ${ }^{1} \mathrm{H}\left(\mathrm{C}_{5} \mathrm{D}_{5} \mathrm{~N}, 300 \mathrm{MHz}\right), \delta$ ppm, (mult.; J em $\mathrm{Hz}$; H): 0,82 (m; H-5), 0,83 (s; H-25), 1,03 (s; H-24), 1,08 (s; H-26), $1,09$ (s; H-27), 1,25 (s; H-23), 1,24 (m; H-12 $\alpha / \mathrm{H}-11 \beta, \mathrm{H}-15 \alpha), 1,37$

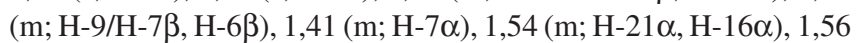
(m; H-6 $\alpha$ ), 1,58 (m; H-22 $\alpha$ ), 1,78 (m; H-18), 1,81 (s; H-30), 1,87 (m;

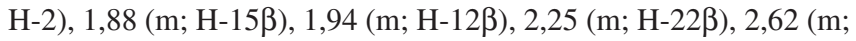
H-16ß), 2,75 (m; H-13), 3,48 (dd; 9,0 e 1,8; H-3), 3,54 (m; H-19), 4,79 (d; 2,4; H-29ß), 4,96 (d; 2,4; H-29 $\alpha$ ). RMN de ${ }^{13} \mathrm{C}\left(\mathrm{C}_{5} \mathrm{D}_{5} \mathrm{~N}\right.$, $75 \mathrm{MHz}) \delta$ ppm: 15,1 (C-27), 16,5 (C-24), 16,6 (C-25/C-26), 18,9 (C-6), 19,6 (C-30), 21,4 (C-11), 26,3 (C-12), 28,4 (C-2), 28,8 (C-23), 30,4 (C-15), 31,4 (C-21), 33,0 (C-16), 35,0 (C-7), 37, 7, 37,8 (H-22), (C-10), 38,9 (C-13), 39,5 (C-1), 39,7 (C-4), 41,3 (C-8), 43,0 (C-14), 47,9 (C-19), 49,9 (C-18), 51,1 (C-9), 56,1 (H-5), 56,8 (C-17), 78,3 (C-3), 110,1 (C-28), 151,5 (C-20), 179,0 (C-29). Esses dados estão de acordo com os encontrados na literatura para o ácido betulínico. ${ }^{16}$

Substância 2: RMN de ${ }^{1} \mathrm{H}\left(\mathrm{CD}_{3} \mathrm{OD}, 300 \mathrm{MHz}\right) \delta$ ppm (mult.; J em Hz; H): 0,74 (s; H-23), 0,84 (s; H-24), 0,94 (s; H-25), 0,95 (s; H-26), 0,99 (s; H-27), 1,00 (s; H-28), 1,68 (s; H-30), 3,17 (dd; 11,1 e 5,4; H-3), 4,58 (dd; 3,6 e 1,8; H-29 $\alpha$ ), 4,70 (dl; H-29ß). RMN de ${ }^{13} \mathrm{C}\left(\mathrm{CD}_{3} \mathrm{OD}, 75 \mathrm{MHz}\right) \delta$ ppm: 15,0 (C-27), 16,1 (C-24), 16,6 (C-26), 16,7 (C-25), 18,4 (C-28), 19,4 (C-30), 19,5 (C-6), 22,0 (C-11), 26,8 (C-12), 28,0 (C-15), 28,6 (C-2), 30,8 (28,0), 31,6 (C-21), 33,3 (C-7), 35,5 (C-16), 38, 1 (C-10) 38,3 (C-13), 39,6 (C-22), 39,9 (C-8), 40,0 (C-4), 41,8 (C-5), 43,2 (C-14), 44,0 (C-17), 48,1 (C-19), 49,8 (C-18), 50,9 (C-9), 79,6 (C-3), 110,1 (C-29), 152,0 (C-20). Esses dados estão de acordo com os encontrados na literatura para o lupeol. ${ }^{17}$

Substância 3: F.M. $\mathrm{C}_{21} \mathrm{H}_{20} \mathrm{O}_{11}, \mathrm{~m} / z$ 447,0852 [M-H], $\mathrm{RMN}$ de ${ }^{1} \mathrm{H}$ $\left(\mathrm{CD}_{3} \mathrm{OD}, 300 \mathrm{MHz}\right) \delta$ ppm (mult.; J em Hz; H): 0,93 (d; 6,0; H-6"), 3,36-3,43 (m; H-5"), 3,34 (d; 9,3; H-4"), 3,73 (dd; 9,0 e 3,3; H-3"), 4,20 (dd; 3,3 e 1,5; H-2"), 5,34 (d; 1,5; H-1"), 6,19 (d; 2,1; H-6), 6,36 (d; 2,1; H-8), 6,90 (d; 8,1; H-5'), 7,28 (dd; 8,1 e 2,1; H-6'), 7,32 (d, 2,1 ; H-2'). RMN de ${ }^{13} \mathrm{C}$ ( $\left.\mathrm{CD}_{3} \mathrm{OD}, 75 \mathrm{MHz}\right) \delta \mathrm{ppm}$ : 17,6 (C-6"), 71,8
(C-2"), 72,0 (C-3"), 72,1 (C-5"), 73,2 (C-4"), 94,7 (C-8), 99,8 (C-6), 103,5 (C-1”), 105,8 (C-10), 116,3 (C-5'), 116,9 (C-2'), 122,8 (C-1'), 136, 2 (C-3), 146,4 (C-3'), 149,7 (C-4'), 158,5 (C-9), 159,3 (C-2), 163,1 (C-5), 165,8 (C-7), 179,6 (C-4). Esses dados estão de acordo com os encontrados na literatura para a quercitrina. ${ }^{18}$

Substância 4: F.M. $\mathrm{C}_{15} \mathrm{H}_{14} \mathrm{O}_{6}, m / z$ 289,0664 [M-H]', RMN de ${ }^{1} \mathrm{H}$ $\left(\mathrm{CD}_{3} \mathrm{OD}, 300 \mathrm{MHz}\right) \delta$ ppm (mult.; J em Hz; H): 2,49 (dd; 16,1 Hz e 8,4 Hz; H-4ax), 2,85 (dd; 16,5 e 8,4; H-4eq), 3,97 (m; H-3), 4,55 (d; 7,5; H-2), 5,85 (d; 2,4 Hz; H-6), 5,92 (d; 2,4; H-8), 6,72 (dd; 7,8 e 1,8; H-6'), 6,74 (d; 7,8; H-5'), 6,82 (d, 1,8; H-2'). RMN de ${ }^{13} \mathrm{C}$ $\left(\mathrm{CD}_{3} \mathrm{OD}, 75 \mathrm{MHz}\right) \delta$ ppm: 28,4 (C-4), 68,7 (C-3), 82,8 (C-2), 95,5 (C-8), 96,3 (C-6), 100,8 (C-10), 115,2 (C-2'), 116,0 (C-5'), 120,0 (C-6'), 132,2 (C-1'), 146,2 (C-3'), 146,2 (C-4'), 156,8 (C-9), 157,5 (C-5), 157,8 (C-7). Esses dados estão de acordo com os encontrados na literatura para a catequina. ${ }^{19,20}$

Substância 5: F.M C ${ }_{21} \mathrm{H}_{20} \mathrm{O}_{12}, m / z$ 463,0800 [M-H]', RMN de ${ }^{1} \mathrm{H}$ $\left(\mathrm{CD}_{3} \mathrm{OD}, 300 \mathrm{MHz}\right) \delta$ ppm (mult.; J em Hz; H): 0,99 (d; 6,0; H-6"), 3,45 (sl; H-4"), 3,54 (d; 2,4; H-5"), 3,65 (sl; H-3"); 4,46 (d; 1,8; H-2"), 5,38 (d; 1,8; H-1"), 6,21 (d; 2, 1; H-6), 6,37 (d; 2, 1; H-8), 7,06 (s; H-2', H-6'). RMN de ${ }^{13} \mathrm{C}\left(\mathrm{CD}_{3} \mathrm{OD}, 75 \mathrm{MHz}\right) \delta \mathrm{ppm}: 17,7$ (C-6"), 71,5 (C-2”), 72,2 (C-5”), 72,3 (C-3”), 73,4 (C-4”), 94,7 (C-8), 99,8 (C-6), 103,5 (C-1'), 106,1 (C-10), 110,4 (C-2'), 110,5 (C-4'), 121,7 (C-1'), 136,2 (C-3), 146,4 (C-2', C-5'), 158,5 (C-9), 159,3 (C-2), 163,2 (C-5), 165,9 (C-7), 179,5 (C-4). Esses dados estão de acordo com os encontrados na literatura para a miricitrina. ${ }^{21}$

\section{Identificação dos compostos fenólicos por CLAE/DAD/EM-EM}

Na Tabela 1 encontram-se listados os compostos identificados nas frações (FA 8 e FA 12) e subfrações (Spx 11, Spx 14, Spx 21 e Spx 37) da fase em acetato de etila do extrato metanólico das folhas de E. flavescens por CLAE acoplada à espectrometria de massas (CLAE/DAD/EM-EM). De acordo com os dados da Tabela 1, pode-se observar que a espécie é uma importante fonte de flavonois, catequinas e ácidos orgânicos. O ácido protocatecuico (6) e o ácido gálico (7) estão presentes na maioria das frações da fase em acetato de etila analisadas, uma vez que as frações Spx vieram das frações FA 6 e FA 7.

A identificação do ácido protocatecuico na fração FA 8 foi realizada com base no pico do cromatograma com tempo de retenção em 4,50 minutos, sendo que o espectro de massas correspondente a esse pico forneceu a massa do respectivo íon molecular desprotonado $\mathrm{m} / \mathrm{z}$ 153 [M - H] e fragmento filho m/z 109, que foram comparados com o padrão, juntamente com dados da literatura. ${ }^{22}$ Pode ser visualizado nos cromatogramas das amostras analisadas, na Figura 2, que houve uma variabilidade nos compostos encontrados. Esta diferença na composição provavelmente pode estar relacionada com a polaridade do eluente utilizado durante o fracionamento e ao tipo de cromatografia empregada.

Para fração FA 12 foram analisados os espectros de massas e as fragmentações $(\mathrm{m} / \mathrm{z})$ dos respectivos íons moleculares desprotonados $[\mathrm{M}-\mathrm{H}]^{-}$e fragmentos $\mathrm{MS}^{2}$ dos picos com tempos de retenção 3,80; 14,$58 ; 15,32$ e 17,99 minutos, o que levou à identificação, por comparação com o padrão e dados da literatura, dos: ácido gálico (7), rutina (8), miricetina (9), quercetina (10) e canferol (11). ${ }^{22-24}$

Nos espectros da fração Spx 11, foram observados os picos dos íons moleculares e seus respectivos fragmentos filhos, das substâncias 7 (ácido gálico), 9 (miricetina), 10 (quercetina) e $\mathbf{1 1}$ (canferol), também encontrados na fração FA 12. Além dessas substâncias, o pico no cromatograma com tempo de retenção em 4,07 minutos apresentou no espectro de massas o pico do íon molecular $m / z, 353[\mathrm{M}-\mathrm{H}]^{-} \mathrm{e}$ os fragmentos filhos $\mathrm{m} / \mathrm{z} 191 \mathrm{e} \mathrm{m} / \mathrm{z}$ 179. Com base nesses dados, foi possível identificar o ácido clorogênico (12) por comparação com o padrão, juntamente com dados da literatura. ${ }^{25}$ 
Os espectros das frações Spx 14 e Spx 21 mostram perfis cromatográficos semelhantes, o que pode ser observado nos cromatogramas na Figura 2. Novamente foram identificados compostos fenólicos semelhantes aos presentes nas frações FA 12 e Spx 11, diferindo apenas pela presença da catequina (4), que foi identificada por apresentar um pico no espectro com tempo de retenção em 4,53 minutos, correspondente no espectro de massas

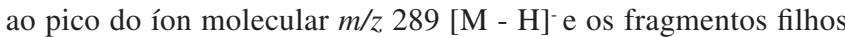
$m / z, 245, m / z, 205$ e $m / z, 179$. A comparação do tempo de retenção e o respectivo espectro de massas com o padrão, juntamente com dados da literatura, permitiu a identificação da catequina nessas duas frações. ${ }^{26}$ Após análise do cromatograma da fração Spx 37, novamente foram identificadas substâncias anteriormente discutidas, além do ácido $p$-cumárico (13), identificado por comparação com o padrão, sendo que o pico com tempo referente ao tempo de retenção em 13,76 minutos apresentou no espectro de massas o pico do íon

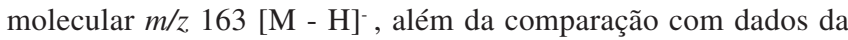
literatura. ${ }^{27}$

\section{Efeitos fitotóxicos sobre a germinação de sementes das fases orgânicas}

Nos bioensaios de germinação das sementes utilizando as fases do extrato metanólico das folhas de $E$. flavescens frente à espécie $M$. pudica, as fases diclorometânica e acetato de etila apresentaram os maiores valores de inibição, com 77,6\% e 71,2\%, respectivamente, enquanto a fase hexânica apresentou $55,2 \%$ e fase $n$-butanólica apresentou $57,0 \%$ de inibição.

Como pode ser observado na Figura 3, para a espécie $S$. obtusifolia as fases orgânicas de E. flavescens apresentaram baixos potenciais de inibição de germinação de sementes, uma vez que a fase hexânica inibiu 3,4\%, a fase DCM inibiu 17,0\%, enquanto as fases AcOEt e $n$-BuOH inibiram $15,0 \%$ e $34,0 \%$, respectivamente.

A presença dos compostos fenólicos, principalmente os flavonoides catequina, miricetina, rutina e quercitrina, identificados na fase AcOEt de E. flavescens, podem explicar os efeitos inibitórios na germinação das sementes de $M$. pudica, uma vez que derivados fenólicos possuem efeitos inibitórios relatados. ${ }^{28}$ Esses compostos provavelmente devem estar presentes também nas fases DCM e $n$-BuOH, uma vez que foram observados altos valores de inibição para essas fases. A espécie invasora $S$. obtusifolia mostrou ser mais resistente quando exposta a ação desses compostos presentes nas fases orgânicas de E. flavescens.

\section{Efeito fitotóxico do triterpeno ácido betulínco e da mistura de flavonoides quercitrina e catequina}

De acordo com os resultados dos experimentos realizados e mostrados na Figura 4, o ácido betulínico estimulou a germinação das sementes de $M$. pudica em $20,0 \%$ e $18,4 \%$ quando testado nas concentrações de 200 e 400 ppm, respectivamente, enquanto que na concentraçãos de 600 ppm o estímulo foi de 14,0\% e, a 800 ppm, houve uma discreta inibição da germinação de sementes (3,0\%). Para a espécie $S$. obtusifolia, houve inibição da germinação das sementes, no entanto, os valores foram extremamente baixos em todas as concentrações testadas, atingindo 5,0\% na concentração de 800 ppm. Outras substâncias triterpenicas, tais como lupeol e lupenona, em estudos anteriores, testadas contra as mesmas plantas-teste, apresentaram valores de inibição extremamente baixos, o mesmo ocorrendo com os triterpenos friedelina e epifriedelinol. ${ }^{29-30}$

Nos bioensaios envolvendo efeitos no alongamento do hipocótilo e radícula, utilizando-se as mesmas plantas-teste, também foram observados baixos potenciais de inibição, o que pode ser visto nas Figuras 5 e 6, respectivamente.

Os maiores potenciais de inibição do crescimento do hipocótilo foram de 15,6\% para S. obtusifolia e 14,0\% para M. pudica, ambos na concentração de 800 ppm. Para o desenvolvimento da radícula, o efeito de inibição mais significativo, nas duas plantas testes, foi observado também na concentração 800 ppm, registrando-se efeitos inibitórios de 14,5\% (M. pudica) e 21,0\% (S. obtusifolia).

Tabela 1. Fenólicos identificados por CLAE/DAD/EM-EM nas frações de E. flavescens

\begin{tabular}{|c|c|c|c|c|}
\hline Fração & Composto fenólico & Tempo de retenção (minutos) & $\begin{array}{c}{[\mathrm{M}-\mathrm{H}]^{-}} \\
\mathrm{m} / \mathrm{z}\end{array}$ & $\begin{array}{l}\mathrm{MS}^{2} \\
\mathrm{~m} / \mathrm{z}\end{array}$ \\
\hline FA 8 & (6) Ác. protocatecuico & 4,50 & 153 & [153] 109 \\
\hline \multirow{6}{*}{ FA 12} & (6) Ác. Protocatecuico & 4,58 & 153 & [153] 109 \\
\hline & (7) Ác. gálico & 3,18 & 169 & [169] 125 \\
\hline & (8) Rutina & 14,58 & 609 & [609] 301, 179 \\
\hline & (9) Miricetina & 15,31 & 317 & {$[317] 179,151$} \\
\hline & (10) Quercetina & 16,43 & 301 & [301] 151 \\
\hline & (11) Canferol & 17,99 & 285 & {$[285] 217,151$} \\
\hline \multirow{5}{*}{ Spx 11} & (7) Ác. gálico & 3,12 & 169 & [169] 125 \\
\hline & (12) Ác. clorogênico & 4,07 & 353 & {$[353] 191,179$} \\
\hline & (9) Miricetina & 15,25 & 317 & {$[317] 179,151$} \\
\hline & (10) Quercetina & 16,49 & 301 & [301] 151 \\
\hline & (11) Canferol & 18,36 & 285 & {$[285] 217,151$} \\
\hline \multirow{5}{*}{ Spx 21} & (7) Ác. gálico & 3,19 & 169 & {$[169] 125$} \\
\hline & (12) Ác. clorogênico & 4,07 & 353 & [353] $191,179,173$ \\
\hline & (4) Catequina & 4,52 & 289 & [289] $245,205,179$ \\
\hline & (8) Rutina & 14,80 & 609 & [609] 301, 179 \\
\hline & (10) Quercetina & 16,55 & 301 & [301] 151 \\
\hline \multirow{7}{*}{ Spx 37} & (7) Ác. gálico & 3,50 & 169 & [169] 125 \\
\hline & (6) Ác. protocatecuico & 4,50 & 153 & [153] 109 \\
\hline & (4) Catequina & 4,54 & 289 & [289] $245,205,179$ \\
\hline & (13) Ác. $p$-cumárico & 13,76 & 163 & [163] 119 \\
\hline & (9) Miricetina & 15,07 & 317 & [317] 179,151 \\
\hline & (10) Quercetina & 16,68 & 301 & [301] 151 \\
\hline & (11) Canferol & 18,05 & 285 & {$[285] 217,151$} \\
\hline
\end{tabular}



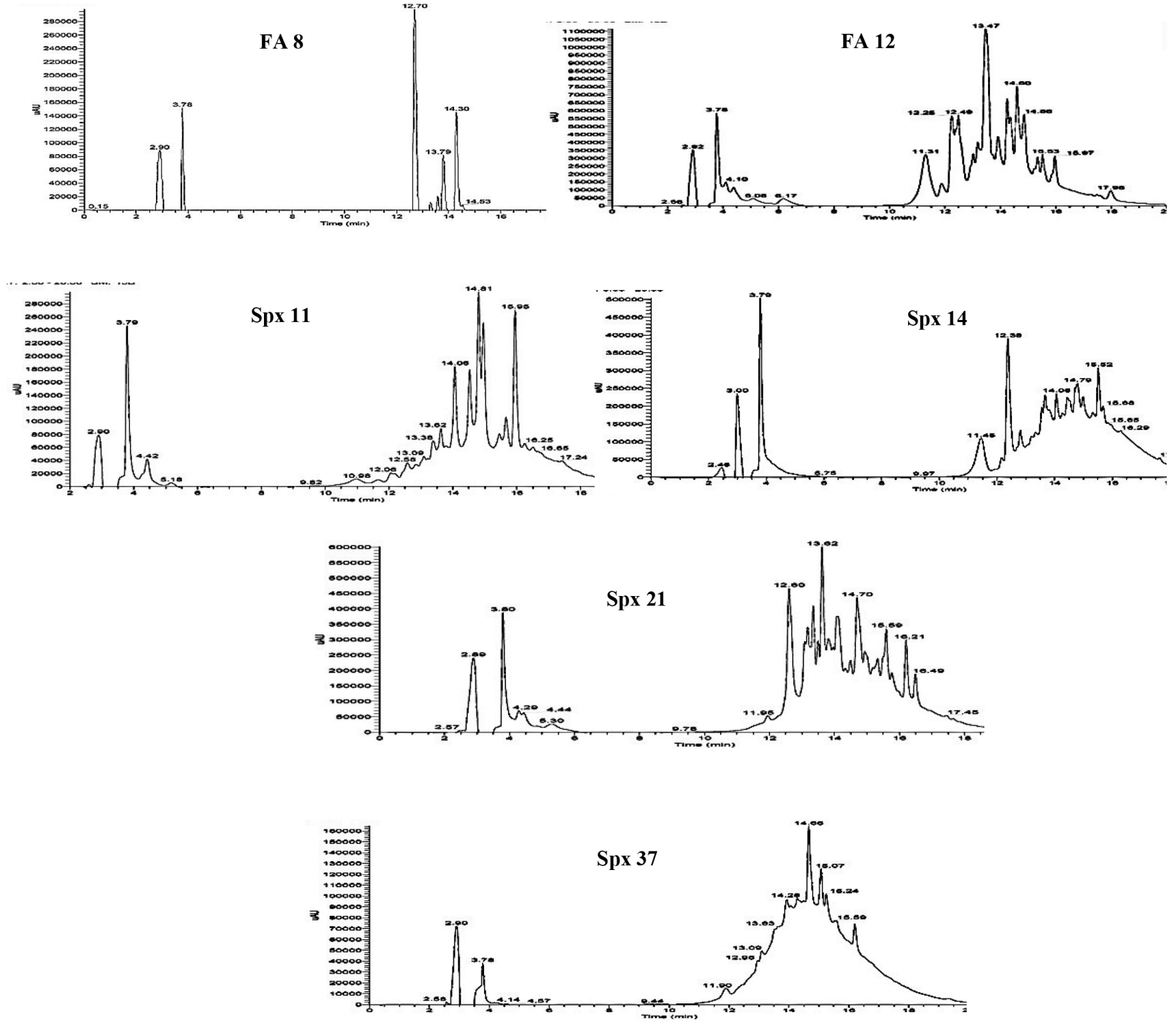

Figura 2. Cromatogramas das frações FA 8, FA 12, Spx 11, Spx 14, Spx 21 e Spx 37

\section{Germinação de sementes}

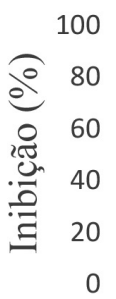
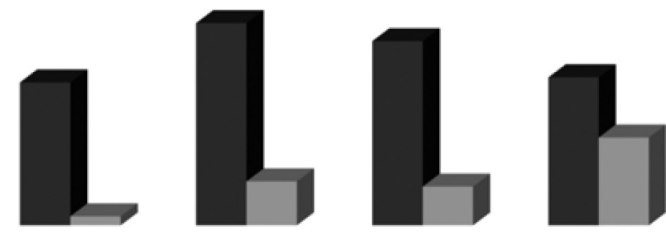

F. HEX
F. AcoEt

F. n-BuOH

\section{Concentração (ppm)}

- M. pudica $\quad$ S. obtusifolia

Figura 3. Potencial de inibição das fases hexânica, diclorometânica, acetato de etila e n-butanólica das folhas de E. flavescens $(1 \% \mathrm{~m} / \mathrm{v})$ sobre a germinação das sementes de M. pudica e S. obtusifolia. Dados expressos em percentual de inibição em relação ao tratamento testemunha, água destilada

Considerando os ensaios de inibição da germinação de sementes e desenvolvimento da radícula e do hipocótilo, embora os valores sejam baixos, $S$. obtusifolia foi mais sensível aos efeitos provocados pelo ácido betulínico (1).

Mesmo com valores modestos de inibição sobre o alongamento da radícula e do hipocótilo, as curvas dos gráficos de regressão polinomial quadrática demonstram também uma relação direta do efeito fitotóxico com o aumento da concentração do ácido betulínico (1), sendo os valores dos coeficientes ajustados para o crescimento do hipocótilo $\left(\mathrm{R}^{2}\right)$ de 0,90 para a espécie $S$. obtusifolia e de 0,96 para $M$. pudica (Figura 5). No caso do alongamento da radícula das mesmas plantas, os valores do coeficiente de determinação ajustados $\left(\mathrm{R}^{2}\right)$ foram de 0,98 e de 0,99 para S. obtusifolia e M. pudica, respectivamente (Figura 6).

O triterpeno lupeol (2), também isolado de E. flavescens, não foi utilizado para os testes de efeito fitotóxico com as mesmas plantas-teste, pois dados encontrados na literatura sobre bioensaios fitotóxicos com triterpenos, entre eles lupeol, mostram que essa substância apresenta baixo efeito fitotóxico, inibindo em aproximadamente 2,0\% 


\section{Efeito fitotóxico sobre a germinação de sementes}

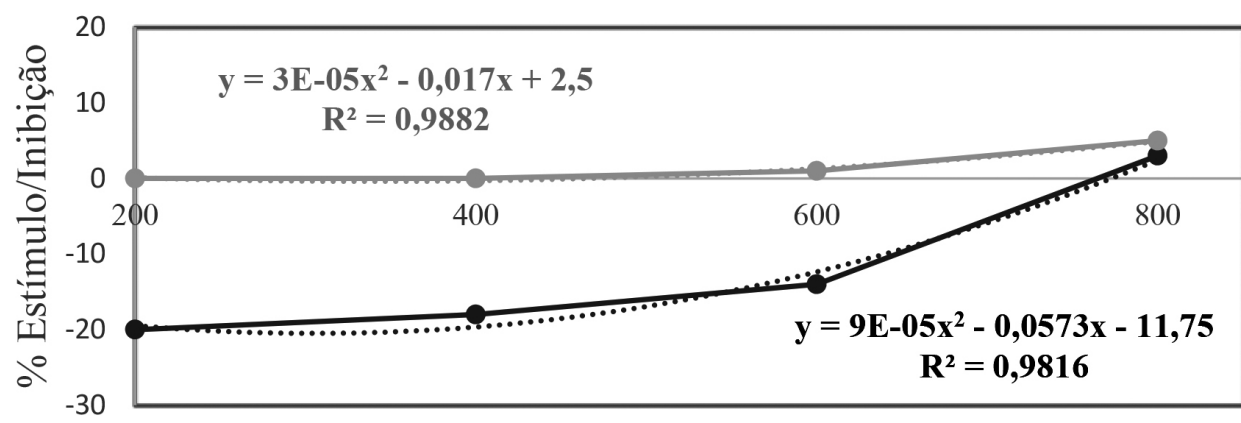

Concentração (ppm)

$\rightarrow$ M. pudica $\longrightarrow$ S. obtusifolia

Figura 4. Potencial de estímulo/inibição do ácido betulínico sobre a geminação de sementes de M. pudica e S. obtusifolia. Dados expressos em percentual de inibição em relação ao tratamento testemunha, água destilada

Efeito fitotóxico sobre o alongamento do hipocótilo

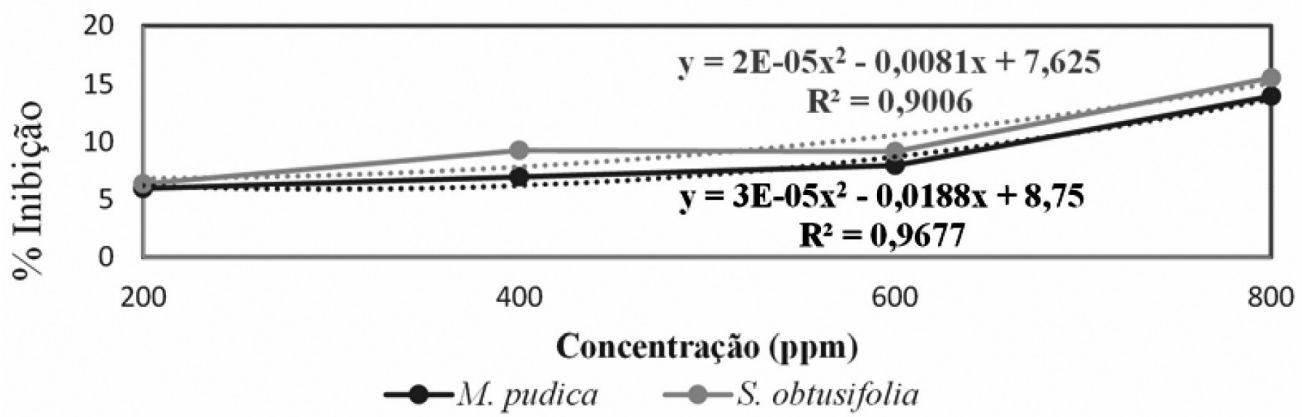

Figura 5. Potencial de inibição do ácido betulínico sobre o alongamento do hipocótilo de M. pudica e S. obtusifolia. Dados expressos em percentual de inibição em relação ao tratamento testemunha, água destilada

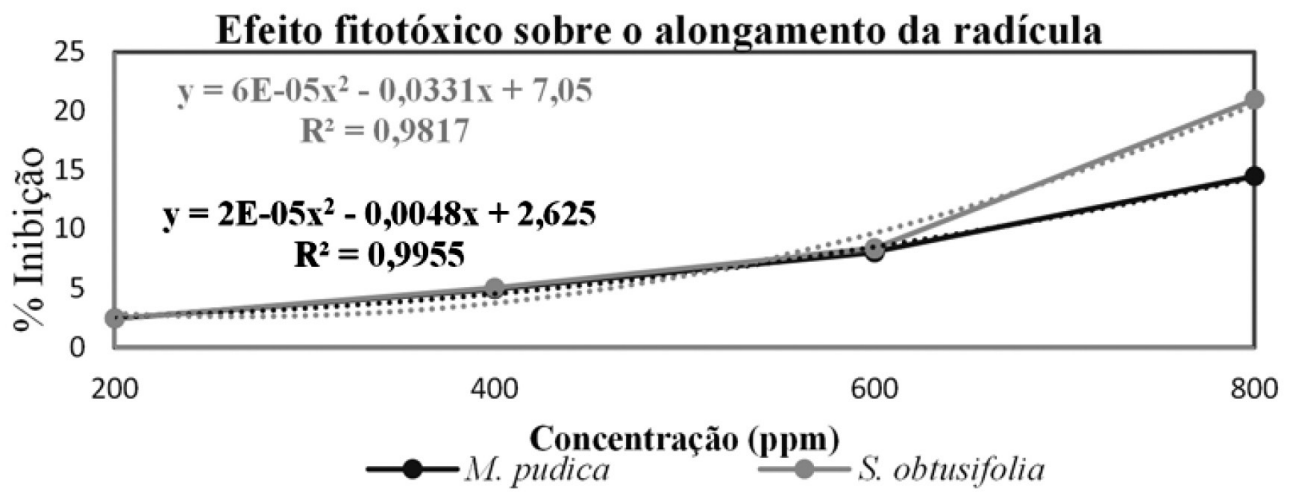

Figura 6. Potencial de inibição do ácido betulínico sobre o alongamento da radícula de M. pudica e S. obtusifolia. Dados expressos em percentual de inibição em relação ao tratamento testemunha, água destilada

a germinação de sementes para ambas as espécies invasoras. Para o alongamento da radícula o triterpeno inibiu em 36,4\% (M. pudica) e $41,3 \%$ (S. obtusifolia), enquanto que para o hipocótilo a inibição foi em torno de $15,0 \%$ frente às mesmas espécies invasoras empregadas nos bioensaios realizados neste trabalho. ${ }^{29}$

Na Tabela 2 são mostrados os efeitos de inibitórios sobre a germinação de sementes e alongamento de plântulas em diferentes concentrações para a mistura (3/4). As equações quadráticas de regressão mostram um tendência não linear de aumento do efeito inibitório com o aumento da concentração da mistura (3/4). Os efeitos de inibição da germinação das sementes de $M$. pudica foram maiores do que sobre as sementes de $S$. obtusifolia nas concentrações testadas, sendo que na concentração de 150 ppm a inibição foi de $92,1 \%$ e
$63,0 \%$, respectivamente, para $M$. pudica e $S$. obtusifolia. A partir das equações da curva no gráfico foi possível calcular o $\mathrm{IC}_{50}$ da mistura (3/4). Para M. pudica o valor do $\mathrm{IC}_{50}$ foi de $32,9 \mathrm{ppm}$, enquanto que para $S$. obtusifolia foi de 69,3 ppm. Esses valores de $\mathrm{IC}_{50}$ evidenciam que a espécie $M$. pudica é mais sensível à mistura de (3/4).

Os resultados obtidos no teste de alongamento da radícula mostraram que a mistura de substâncias $\mathbf{3}$ e $\mathbf{4}$ apresentou relevantes potenciais de inibição frente às duas plantas daninhas nas concentrações testadas. Os melhores resultados de inibição foram nas concentrações 100 e 150 ppm, nas quais a mistura inibiu em 60,8 e $74,5 \%$, respectivamente, o desenvolvimento da radícula da espécie M. pudica. Já para a espécie $S$. obtusifolia os percentuais de inibição foram maiores comparados a $M$. pudica em todas as concentrações. 
Tabela 2. Potencial de inibição da mistura quercitrina e catequina sobre a germinação das sementes e desenvolvimento da radícula e do hipocótilo de $M$. pudica e S. obtusifolia

\begin{tabular}{|c|c|c|c|c|c|c|}
\hline \multirow{3}{*}{$\begin{array}{l}\text { Concentração } \\
\text { [ppm] }\end{array}$} & \multicolumn{6}{|c|}{ Valores inibitórios (\%) } \\
\hline & \multicolumn{3}{|c|}{ M. pudica } & \multicolumn{3}{|c|}{ S. obtusifolia } \\
\hline & Germ. & Rad. & Hip. & Germ & Rad. & Hip. \\
\hline 50 & 55,8 & 32,5 & 40,7 & 8,0 & 41,0 & 4,4 \\
\hline 75 & 58,0 & 33,0 & 52,7 & 10,0 & 52,9 & 15,8 \\
\hline 100 & 73,7 & 60,8 & 59,3 & 16,7 & 74,5 & 18,5 \\
\hline 150 & 92,1 & 72,5 & 78,8 & 63,0 & 73,6 & 76,1 \\
\hline $\mathrm{IC}_{50}[\mathrm{ppm}]$ & 32,9 & 90,7 & 72,3 & 69,3 & 62,9 & 132,2 \\
\hline $\mathrm{R}^{2}$ & 0,96 & 0,88 & 0,99 & 0,99 & 0,93 & 0,98 \\
\hline
\end{tabular}

Nas concetrações de 100 e 150 ppm, foi possível verificar inibição de 74,5 e $73,6 \%$, respectivamente. Ao contrário do teste para a inibição da germinação de sementes frente às plantas-teste, o cálculo do $\mathrm{IC}_{50}$ revela uma maior sensibilidade da espécie $S$. obtusifolia $(62,9 \mathrm{ppm})$ em relação à espécie $M$. pudica $(90,7 \mathrm{ppm})$ para o alongamento da radícula quando expostas a mistura testada.

Para o teste de inibição do alongamento do hipocótilo, a mistura de substâncias também apresentou relevantes potenciais de inibição frente às mesmas plantas-teste. A Tabela 2 mostra que os valores de inibição mais altos foram encontrados nas concentrações de 100 e 150 ppm, nas quais a mistura inibiu 59,3 e 78,0\%, respectivamente, o alongamento do hipocótilo da espécie $M$. pudica. Já para a espécie S. obtusifolia o percentual de inibição mais relevante foi de $76,1 \%$, na concentração testada de $150 \mathrm{ppm}$. Novamente, valores dos coeficientes ajustados, $\left(\mathrm{R}^{2}\right)$ 0,99 para M. pudica e $\left(\mathrm{R}^{2}\right)$ 0,98 para o S. obtusifolia evidenciam uma tendência não linear para o alongamento das plântulas. $\mathrm{O} \mathrm{IC}_{50}$ para as espécies $M$. pudica $(72,3 \mathrm{ppm})$ e $S$. obtusifolia (132,2 ppm) mostram maior sensibilidade da espécie $M$. pudica em relação à espécie $S$. obtusifolia, quando expostas à mistura testada.

Nos testes de efeitos fitotóxicos realizados na mistura quercitrina e catequina os altos valores de inibição podem estar associados ao efeito sinérgico da mistura, pois os valores de $\mathrm{IC}_{50}$ foram menores para as duas espécies invasoras, quando comparados com testes realizados com a catequina (4) em estudos anteriores com as mesmas plantas teste. ${ }^{31}$ Para a substância (3) também são relatados eficientes efeitos fitotóxicos frente a inibição de germinação de sementes e alongamento da radícula e do hipocótilo da espécie Lactuva sativa. ${ }^{32}$

\section{CONCLUSÃO}

Foi realizado o primeiro estudo químico com as fases orgânicas das folhas de E. flavescens. Esta investigação mostrou que a espécie E. flavescens é uma importante fonte de triterpenos, flavonoides e ácidos fenólicos, classe de substâncias geralmente associados ao gênero Eugenia.

O estudo do efeito fitotóxico mostrou que as fases orgânicas DCM e AcOEt apresentaram maiores percentuais de inibição da germinação de sementes frente as plantas testadas, sendo que a espécie $M$. pudica mostrou-se mais sensível em todas fases testadas.

No bioensaio com o triterpeno ácido betulínico, os testes mostraram baixos valores de inibição para as duas plantas-teste, não ultrapassando 35,0\% de inibição. Para a mistura de flavonoides quercitrina e catequina foram observados potentes efeitos fitotóxicos de inibição nas concentrações testadas. A presença de substâncias fenólicas em E. flavescens pode explicar esse efeito na espécie, uma vez que compostos fenólicos tendem a exercer efeitos inibitórios na germinação e crescimento de plântulas.
Portanto, esse estudo indica que as fases orgânicas das folhas da espécie vegetal $E$. flavescens possuem efeito fitotóxico e podem ser utilizadas na composição de bioherbicidas no controle das plantas invasoras testadas nessa investigação.

\section{MATERIAL SUPLEMENTAR}

Todos os espectros de RMN e de EMAR e os cromatogramas, assim como os gráficos de efeitos fitotóxicos utilizados neste trabalho, estão disponíveis em http://quimicanova.sbq.org.br, na forma de arquivo PDF, com acesso livre.

\section{AGRADECIMENTOS}

Ao PPGQ, à PROPESP, ao ICEN-UFPA e à CAPES, pelo apoio financeiro, e à EMBRAPA, pelo suporte e infraestrutura.

\section{REFERÊNCIAS}

1. Magina, M. D. A.; Dalmarco, E. M.; Dalmarco, J. B.; Quim. Nova 2012, 35,1184 .

2. Reynertson, K. A.; Yang, H.; Jiang, B.; Basile, M. J.; Kennelly, E. J.; Food Chem. 2008, 109, 883.

3. Magina, M. D. A.; Dalmarco, E. M; Wisniewski Jr., E.; Simionatto, E. L.; Dalmarco, J. B.; Pizzolatti, M. G.; Brighente, I. M. C.; J. Nat. Med. 2009, 63, 345 .

4. Cole, R. A.; Haber, W. A.; Setzer, W. N.; Biochem. Syst. Ecol. 2007, 35, 877.

5. Silva, N. C. F.; Resumos do $23^{\circ}$ Seminário de Iniciação Científica da UFPA, Belém, 2012.

6. Inoue, M. H.; Santana, D. C.; Souza Filho, A. P. S.; Possamai, A. C. S.; Silva, L. E.; Pereira, M. J. B.; Pereira, K. M.; Planta Daninha 2010, 28, 489.

7. Soares, W. L., Porto, M. F.; Ciência e Saúde Coletiva 2007, 12, 131.

8. Bhadoria, P. B. S.; Am. J. Exp. Agric. 2011, 1, 7.

9. Novakova, L.; Spáčil, Z.; Seifrtová, M.; Opletal, L.; Solich, P.; Talanta 2010, 80, 1970.

10. Inderjit; Dakshini, K. M. M.; Bot. Rev. 1995, 6, 28.

11. Macías, F. A.; Castellano, D.; Molinillo, J. M. G.; J. Agric. Food Chem. 2000, 48,2512

12. Ministério da Agricultura, Pecuária e Abastecimento; Regras para análise de sementes, MAPA: Brasília, 2009.

13. Souza Filho, A. P. S.; Dutra, S.; Silva, M. A. M. M.; Planta Daninha 1998, 16, 2.

14. Santos, L. S.; Borges, F. C.; Oliveira, M. N.; Ferreira, I. C. S.; Guilhon, G. M. S. P.; Souza Filho, A. P. S.; Santos, A. S.; Arruda, M. S. P.; Müller, A. H.; Arruda, A. C.; Allelopathy Journal 2007, 20, 235. 
15. Wang, G.; Yin, W.; Zhou, Z. Y.; Hsieh, K. L.; Liu, J. K.; J. Asian Nat. Prod. Res. 2010, 12, 770.

16. Cichewicz, R. H.; Kouzi, S. A.; Med. Res. Rev. 2004, 24, 90.

17. Shakeri, A.; Ahmadian, M.; International Journal of Farming and Allied Sciences 2014, 3, 1065.

18. Hasan, S.; Ahmed, I.; Mondal, S.; Masud, M. M.; Sadhu, S. K.; Ishibashi, M.; Uddin, S. J.; Orient. Pharm. Exp. Med. 2006, 6, 355.

19. Benavides, A.; Montoro, P.; Bassarello, C., Piacente, S.; Pizza, C.; J. Pharm. Biomed. Anal. 2006, 40, 639.

20. Cintra, P.; Malaspin, O.; Petacci, F.; Fernandes, J. B.; Bueno, O. C.; Vieira, P. C.; Silva, M. F. G. F.; J. Braz. Chem. Soc. 2002, 13, 115.

21. Aderogba, M. A.; Ndhlala, A. R.; Rengasamy, K. R.; Van Staden, J.; Molecules 2013, 18, 12633.

22. Fabre, N.; Rustan, I.; Hoffmann, E.; Quetin-Leclercq, J.; J. Am. Soc. Mass Spectrom. 2001, 12, 707.

23. Caligari, P. D. S.; Simirgiotis, M. J.; Schemeda-Hirschman, G.; Food Chem. 2009, 115, 775.

24. Saldanha, L. L.; Vilegas, W.; Dokkedal, A. L.; Molecules 2013, 18, 8402.
25. Pearson, J. L.; Lee, S.; Suresh, H.; Low, M.; Nang, M.; Singh, S.; Lamin, F.; Kazzem, M.; Sullivan, S.; Khoo ,C. S.; ISRN Anal. Chem. 2014.

26. Bravo, M. N.; Silva, S.; Coelho, A.V.; Vilas Boas, L.; Bronze, M. R.; Anal. Chim. Acta 2006, 563, 84.

27. Plazonic, A.; Bucar, F.; Maleš, Ž.; Mornar, A; Nigovic, B; Kujundžić, N.; Molecules 2009, 14, 2466.

28. Santos, S. I. Moraes, M. L. L; Rezende, M. O. O.; Souza Filho, A. P. S.; Ecletica Quim. 2011, 36, 51.

29. Souza-Filho, A.P.S.; Santos, R. A.; Santos, L. S.; Guilhon, G. M.P.; Santos, A. S.; Souza Filho, A. P. S.; Guilhon, G. M. S. P.; Santos, L. S.; Planta Daninha 2010, 28, 689.

30. Santos, L. S.; Santos, J. C. L.; Souza Filho, A. P. S.; Corrêa, M. J. C.; Veiga, T. A. M.; Freitas, V. C. M.; Ferreira, I. C. S.; Gonçalves, N. S.; Silva, C. E.; Guilhon, G. M. S. P.; Planta Daninha 2008, 26, 531.

31. Lobo, L. T.; Castro, K. C. F.; Arruda, M. S. P.; Silva, M. N.; Arruda, A. C.; Müller, A. H.; Arruda, G. M. S. P.; Santos, A. S.; Souza Filho, A. P. S.; Quim. Nova 2008, 31, 493.

32. Almeida, L. F. R.; Tese de Doutorado, Universidade Estadual Paulista, Brasil, 2006. 